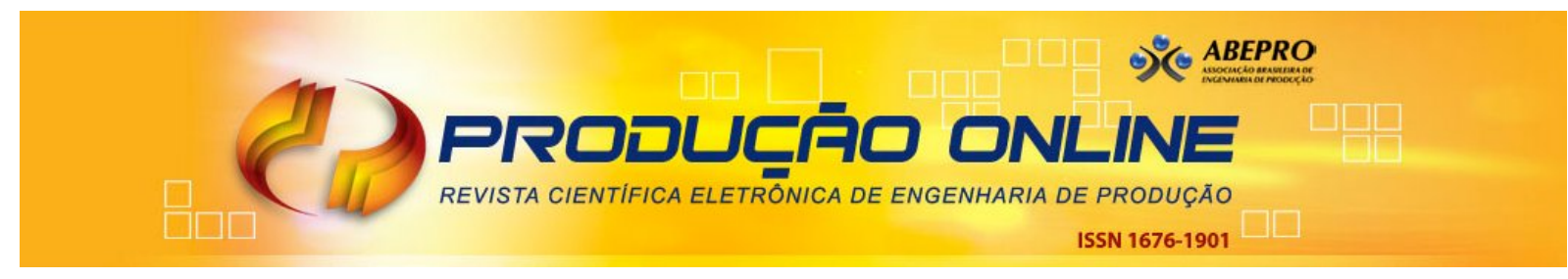

\title{
DIAGNÓSTICO DO GERENCIAMENTO DA CADEIA DE SUPRIMENTOS EM UM RESTAURANTE UNIVERSITÁRIO ATRAVÉS DE ESTUDO EXPLORATÓRIO
}

\section{ANALYSIS OF SUPPLY CHAIN MANAGEMENT IN A UNIVERSITY RESTAURANT THROUGH EXPLORATORY STUDY}

\author{
Anna Paula Galvão Scheidegger* E-mail: anninha pgs@hotmail.com \\ Juliana Helena Daroz** E-mail: ju gaudencio@hotmail.com \\ Fabio Favaretto** E-mail: fabio.favaretto@unifei.edu.br \\ Renato da Silva Lima** E-mail: rslima@unifei.edu.br \\ * Texas A\&M University \\ **Universidade Federal de Itajubá
}

\begin{abstract}
Resumo: Ganha cada vez mais relevância o setor de serviços na economia e sociedade brasileira. No entanto, os desafios das empresas, em especial micro e pequenas empresas, são inúmeros quando se considera a competitividade e as dificuldades impostas pela estrutura de mercado brasileira. Desta forma, compreender o processo de gerenciamento da cadeia de suprimentos de pequenas empresas torna-se extremamente importante, pois pode contribuir para a sobrevivência das mesmas. Por isso, o presente trabalho tem como objetivo realizar um diagnóstico do processo de gerenciamento da cadeia de suprimentos dentro do setor de serviços. Para isso, adotou-se como objeto de estudo um restaurante universitário, uma vez que o mesmo além de enfrentar os usuais desafios das micro empresas, apresenta características especiais que tornam o processo ainda mais complexo. De caráter exploratório e utilizando-se de pesquisa qualitativa, o trabalho desenvolveu, através de ferramentas de mapeamento como o SIPOC e análise SWOT, uma melhor compreensão sobre o contexto e identificou algumas oportunidades para futuros estudos mais profundos, cumprindo assim com os objetivos propostos.
\end{abstract}

Palavras-chave: Gerenciamento da cadeia de suprimentos. Gestão da informação. Fluxo de suprimentos. Setor de serviços. Restaurante universitário.

Abstract: The service sector has been gaining increasing relevance in the Brazilian economy and society. However, the challenges faced by the enterprises, especially small and micro enterprises, are numerous when considering the competitiveness and the difficulties imposed by the Brazilian market structure. Thus, understanding the supply chain management of small business becomes extremely important, once it can contribute to their survival. Therefore, this study aims to conduct an assessment of the supply chain management process within the service sector. For this, it was adopted as object of study a university restaurant, which while face the usual challenges of micro enterprises, also has special characteristics that make the process even more complex. With exploratory character and using qualitative research, the work developed through mapping tools such as SIPOC and SWOT analysis, a better understanding of the context and identified some opportunities for further study, thus complying with the proposed objectives.

Keywords: Supply chain management. Information management. Supply flow. Service sector. University restaurant.

Revista Produção Online, Florianópolis, SC, v.15, n. 1, p. 375-402, jan./mar. 2015. 


\section{INTRODUÇÃO}

Discussões sobre o gerenciamento da cadeia de suprimentos têm aumentado consideravelmente desde a década de 80 , quando as empresas começaram a vislumbrar os benefícios de relações colaborativas dentro e além de suas próprias organizações (LUMMUS e VOKURKA, 1999). Entretanto, observa-se que o foco principal, tanto de estudiosos quanto de empresários, ainda está no ramo industrial. Assim, há ainda muito para ser explorado sobre este contexto no setor de serviços, principalmente no que se refere a organizações-laboratórios de empreendedorismo.

Segundo o Ministério de Desenvolvimento, Indústria e Comércio Exterior (2010), o setor terciário (de prestação de serviços) vem ganhando a atenção de investidores e governos no Brasil e no mundo. No ano de 2009, o setor respondeu por $68,5 \%$ do Produto Interno Bruto (PIB) e por mais de $70 \%$ dos empregos formais no Brasil. Tais dados indicam a relevância do setor de serviços para a economia e a sociedade brasileira. Conforme dados do Instituto Brasileiro de Geografia e Estatística (2008), dentro do setor de serviços, os serviços de alimentação foram responsáveis pela absorção da maior parte do pessoal ocupado (1,2 milhão) e correspondem a 22,7\% do total das empresas de serviços no Brasil. Assim, a relevância do setor, bem como a importância da busca por um melhor desempenho para a sobrevivência do mesmo, como indicado por Torres Júnior e Miyake (2011) e Torres Júnior e Lopes (2013), justificam a oportunidade de pesquisa explorada por este estudo.

O presente estudo tem como objetivo diagnosticar o processo de gerenciamento da cadeia de suprimentos dentro de um restaurante acadêmico, através de ferramentas de mapeamento, para identificar oportunidades de futuras pesquisas neste ambiente.

\section{FUNDAMENTAÇÃO TEÓRICA}

\subsection{Cadeia de suprimentos}

Segundo Ganeshan e Harrison (1995), a cadeia de suprimentos é uma rede de instalações e responsável pelas funções de aquisição de materiais, Revista Produção Online, Florianópolis, SC, v.15, n. 1, p. 375-402, jan./mar. 2015. 
transformação destes materiais em produtos semi-acabados e acabados, e a distribuição destes produtos aos consumidores finais. A cadeia de suprimentos existe tanto nas indústrias quanto nas empresas de serviços, porém com complexidade que varia de cadeia para cadeia.

\subsubsection{Gerenciamento da cadeia de suprimentos}

Segundo Ballou (2004), o gerenciamento da cadeia de suprimentos (GCS) destaca as interações logísticas que ocorrem entre as funções de marketing, logística e produção no âmbito de uma empresa, e dessas mesmas interações entre as empresas legalmente separadas no âmbito do fluxo de produtos.

Os autores Simchi-Levi, Kaminsky e Simchi-Levi (2003) definem a gestão da cadeia de suprimentos como um conjunto de abordagens utilizadas para integrar eficientemente fornecedores, fabricantes, depósitos e armazéns, de forma que a mercadoria seja produzida e distribuída na quantidade certa, para localização certa e no tempo certo, de forma a minimizar os custos globais do sistema ao mesmo tempo em que atinge o nível de serviço desejado.

O gerenciamento da cadeia de suprimentos envolve, portanto, considerar sistematicamente as inter-relações entre os processos, bem como o nível de interação entre as organizações que compõem a cadeia, já que a colaboração, a integração e cooperação entre os elos são princípios fundamentais (FREITAS et al., 2010). Assim, para Ballou (2004), uma boa gestão da cadeia de suprimentos pode aumentar as vendas e não apenas reduzir custos. Um dos principais benefícios que se espera em uma cadeia de suprimentos é o correto nível de estoque nas empresas (DROHOMERETSKI e FAVARETTO, 2013) e a diminuição no tempo de espera das empresas clientes (GUT e BELFIORE, 2009).

\subsubsection{Gerenciamento da informação nas cadeias de suprimentos}

O fluxo de materiais em uma cadeia de suprimentos, dos fornecedores para os consumidores, é coordenado por um fluxo de informações em sentido contrário. Janak (1996) destaca de forma incisiva a necessidade deste fluxo, da mesma forma 
que o fluxo de materiais. No final da década de 90 , esta ideia foi consolidada nas pesquisas acadêmicas e também na prática, devido, em parte, ao crescimento do uso de sistemas integrados de informação como os sistemas ERP (Enterprise Resources Planing). Zeng et al. (2003) sustentam que estes sistemas suportam e permitem a integração e o fluxo de informações dos processos de negócios de uma empresa.

Essas interpretações confirmam o consenso de que o gerenciamento da cadeia de suprimentos tem dois grandes focos: o fluxo de materiais e o fluxo de produtos (PIRES e CARRETERO-DIAZ, 2007). Outros autores mostram mais fluxos, como Berrah e Cliville (2007) que afirmam que uma cadeia de suprimentos é composta pelos fluxos de materiais, de informações, financeiros e de conhecimento, todos com o objetivo de satisfazer as necessidades dos usuários finais. Sahin e Robinson (2002) apontam a cadeia de suprimentos como fluxos financeiros, de materiais e de informações. O fluxo de informações é um direcionador (driver) que influencia os demais direcionadores de uma cadeia e, por isto tem muita importância para o seu desenvolvimento (ROSSIN, 2007).

Em sua pesquisa, Gundlach et al. (2006) asseveram que os trabalhos sobre o assunto apresentam algumas diversidades, porém entre os pontos comuns, pode-se destacar a necessidade de integração entre os elos de uma cadeia, obtida através de um intensivo fluxo de informações. Neste sentido, Lin et al. (2006) enfatizam a disponibilidade de tecnologias para troca de dados em tempo real como um real, habilitador da gestão de uma cadeia de suprimentos.

O papel das informações também é destacado por Lewis e Talayevsky (2004), como o coordenador de uma cadeia de suprimentos e consideram este fluxo como fator de melhoria dos custos de transação e dos resultados gerais de uma cadeia. Childerhouse et al. (2003a) concebem o fluxo de informações como um habilitador de melhorias em uma cadeia de suprimentos automotiva.

Para Sahin e Robinson (2002), o principal objetivo do fluxo de informações em uma cadeia de suprimentos é a coordenação dos fluxos físicos. Este deve fluir entre todos os elementos da cadeia e não somente entre os principais fabricantes e os pontos de venda ou fornecedores imediatos. Entre as informações que fazem parte deste fluxo, podem ser destacadas: 
- Posição de estoque;

- Quantidade a ser movimentada;

- Desempenho do sistema (custos, rotação de estoques e outros indicadores); e,

- Dados de planejamento da produção (previsões, consumo, dados para reposição, tamanhos de lotes, tempos e restrições de capacidade).

As principais informações que compõem o respectivo fluxo em uma cadeia de suprimentos são aquelas relacionadas às entregas de produtos (desde a matériaprima até os produtos finais), aos fluxos de materiais, de estoques, e de ordens de produção (CHILDERHOUSE et al., 2003b). As informações relacionadas à demanda de uma cadeia são destacadas por Gavirneni (2002), que as vê como um dos principais fluxos de cadeias onde existem restrições de capacidade.

A troca de grandes volumes de informações entre os elos de uma cadeia é possível graças ao uso da tecnologia da informação (TI). Estas trocas são praticamente inviáveis em formatos não-eletrônicos, devido ao grande volume, nível de detalhamento e questões, como disponibilidade e armazenamento.

Lin e Tseng (2006) analisam pesquisas anteriores sobre o assunto e concluem que a aplicação efetiva de sistemas de informação estabelece uma competitividade superior à uma cadeia de suprimentos. O papel do uso da tecnologia da informação como diferencial competitivo também é destacado por McLaughlin et al. (2003), que analisam, em um estudo de caso, a utilização de sistemas para a otimização e o planejamento de transportes, relacionando direta e proporcionalmente esse emprego aos resultados positivos obtidos.

Procurou-se até aqui mostrar a importância do fluxo de informações em uma cadeia de suprimentos. A seguir serão apresentados alguns conceitos sobre a gestão deste fluxo de informações.

Uma iniciativa, uma técnica ou um método aplicados em determinada situação podem ser chamados de "prática". Na gestão da cadeia de suprimentos, algumas práticas são consolidadas como, por exemplo, o outsourcing e o planejamento colaborativo. O objetivo desta seção é destacar as práticas relacionadas à gestão de informações na cadeia de suprimentos, com destaque para o compartilhamento ou troca de informações entre as empresas de uma cadeia. 
Em uma pesquisa para medir a importância do gerenciamento e compartilhamento de informações, Zhou e Benton Jr. (2007) concluem que a efetividade de uma cadeia de suprimentos e o compartilhamento de informações são diretamente proporcionais. Assim, as cadeias mais efetivas são aquelas que gerenciam melhor e mais compartilham informações entre seus elos.

Sahin e Robinson (2002) apresentam uma extensa pesquisa sobre o compartilhamento de informações e o fluxo de coordenação em uma cadeia de suprimentos. Entre as conclusões apontadas destaca-se o compartilhamento de informações em qualquer grau (parcial ou total) como benéfico para a cadeia e que isso requer um bom gerenciamento deste fluxo. Os autores afirmam que os habilitadores do compartilhamento são a associação entre técnicas para troca de informações e métodos de gestão.

Outra prática a ser citada na gestão de informações é a utilização e integração de sistemas de planejamento das necessidades das empresas ou sistemas integrados de gestão (Enterprise Resources Planing - ERP). Apesar de não ser uma prática diretamente relacionada à gestão da cadeia de suprimentos, Sahin e Robinson (2002) destacam que muitas empresas focais, ou que podem controlar uma cadeia de suprimentos dispõem destes sistemas mas não utilizam todo potencial disponível para compartilhar informações.

A qualidade da informação e o seu gerenciamento estão despontando no meio acadêmico e empresarial como uma prática benéfica em diversas situações. Seus resultados podem ser expandidos para a cadeia de suprimentos, levando-se em conta o grande volume de informações que devem ser gerenciadas. Uma pesquisa apresentada por Rossin (2007) indica que os efeitos de fluxos com baixa qualidade da informação podem gerar resultados ruins em estoques, custos e serviços ao consumidor.

Em outra pesquisa sobre a qualidade da informação, Forslund e Jonsson (2007) destacam sua importância nas informações utilizadas para se fazer as previsões de uma cadeia de suprimentos, considerando a boa qualidade destas informações como fator fundamental para a competitividade da cadeia. Os autores afirmam que somente a disponibilidade dessas informações não é suficiente, sendo que a confiabilidade, a temporalidade e a acuracidade devem ser consideradas. 
Também apontam que, devido à importância desse assunto, novas pesquisas devem ser desenvolvidas para verificar o amplo impacto da qualidade das informações em cadeias de suprimentos.

Em termos da generalização das aplicações, Kärkkäinen et al. (2003) afirmam que as práticas de gestão da informação em redes de suprimentos são baseadas em grandes soluções genéricas, que não são adequadas a empresas com particularidades complexas e específicas. Neste sentido, propõem um gerenciamento da informação baseado no gerenciamento de produtos (softwares e sistemas de informação) customizados e não-padronizados. Com isto pode-se encontrar uma solução específica para cada necessidade de gerenciamento da informação, mesmo nos níveis mais detalhados de cada empresa em particular.

De uma forma geral, a gestão da informação em uma cadeia de suprimentos é apontada como algo essencial. Schnetzler e Schönsleben (2007) apontam que a gestão da informação tem um impacto positivo em todas as áreas de uma cadeia de suprimentos: qualidade, confiabilidade na entrega, tempos de entrega, flexibilidade e custos.

Esta seção destacou a gestão da informação e suas práticas em cadeias de suprimentos de produtos manufaturados. A seguir serão apresentados conceitos sobre a gestão de informação em empresas essencialmente prestadoras de serviços.

\subsection{Gestão da informação no setor de serviços}

O setor de serviços é um segmento econômico que está em constante crescimento e desenvolvimento, sendo grande gerador de emprego e renda (FREITAS, 2005). No geral, bens podem ser definidos como objetos, dispositivos ou coisas, ao passo que serviços podem ser definidos como ações, esforços ou desempenhos consistindo em uma série de atividades mais ou menos intangíveis que, normalmente, mas não necessariamente ocorrem nas interações entre o cliente e os funcionários de serviço (GRÖNROOS, 2004).

Ballou (2004) afirma que apesar de muitas companhias como fast foods, editora de jornais e varejo de mercadorias serem consideradas como empresas de 
serviço, elas, na verdade, fabricam um produto. Conforme Classificação Nacional de Atividades Econômicas proposta pelo Instituto Brasileiro de Geografia e Estatística (2007), as empresas de serviços de alimentação têm como característica o preparo de refeições e bebidas para consumo imediato, com ou sem serviço completo e a preparação de alimentos por encomenda. Compreendendo assim: restaurantes, bares, lanchonetes, cantinas, quiosques e trailers.

Em uma perspectiva histórica da gestão da informação na prestação de serviços, pode-se citar Edvinsson (1992), que em seu trabalho sobre forças críticas para liderança no setor de serviços, destaca o papel da informação e de seu gerenciamento. O autor mostra o movimento em direção ao capital intelectual (consolidado na Década de 90), que necessariamente deve ser suportado por uma tecnologia que permita a troca e o armazenamento de informações, assim como permita sua recuperação de forma rápida e simples, para garantir o acesso ao conhecimento. Nesta mesma linha, Berkley e Gupta (1995) afirmam que para uma empresa prestadora de serviços obter qualidade em seus serviços ela deve ter uma boa capacidade para coletar, processar e distribuir informação. Fechando esta perspectiva histórica, Sigala e Christou (2006) afirmam no Editorial do volume especial em Tendências Globais e Desafios em Serviços do periódico Managing Service Quality que os dois principais desafios para estas empresas é o gerenciamento da informação (e sua tecnologia) e do conhecimento.

Em Westerback (2000) são identificadas as melhores práticas de gestão da informação em órgãos do governo federal dos EUA, predominantemente prestadores de serviços. As práticas chave identificadas na pesquisa são:

- Uso de medidas de desempenho para identificar o retorno sobre o investimento;

- Tomar partido das tecnologias de ponta; e,

- Usar técnicas comprovadas de gestão de projetos.

As práticas de gestão da informação aplicadas na gestão das cadeias de suprimentos de produtos devem ser adaptadas para as cadeias de serviços, segundo Chou et al. (2006), devido às suas características e necessidades específicas. Estas adaptações estão prosperando devido à crescente importância da participação dos serviços nas receitas de empresas e governos. 
A necessidade de um bom gerenciamento de dados e informações em qualquer tipo de empresa ou situação é relatada por Swartz (2007), pois a informação é o maior bem de uma empresa. Ainda segundo o autor, a maioria das organizações de serviços não faz este gerenciamento de forma adequada, gerando e expandindo problemas decorrentes e apresenta um checklist para o seu bom gerenciamento.

Devido à natureza intrínseca das empresas prestadoras de serviços, a aplicação de práticas orientadas às estas deve considerar a diferença com as que produzem bens, tais como: execução na presença do cliente, intangibilidade e impossibilidade de armazenamento. Brady e Fellenz (2007) afirmam que, apesar do recente destaque que os serviços vêm recebendo de uma forma geral, as tecnologias de comunicação e informação ainda são em sua maioria orientadas para a produção de bens.

Para De Waart e Kemper (2004), mesmo em cadeias de suprimentos industriais, o componente de serviços é inseparável, mesmo que seja somente para o relacionamento pós-venda com os clientes. Os autores apontam que só recentemente as empresas têm colocado esta prestação de serviços como um negócio e que este pode ser rentável, indicando ações para que isto possa ser alcançado. Ruggles (2005) também aponta diferenças entre as cadeias de suprimentos industriais e as de prestação de serviços, indicando que estas últimas apresentam grande potencial de geração de rendimentos.

Por se tratar de um assunto recente nos campos da pesquisa e prática (CHOU et al., 2006 e BALTACIOGLU, 2007), não existem modelos gerais largamente aceitos. Os modelos de cadeias de suprimentos são, em sua maioria, orientados a produtos, e que apenas recentemente começaram a aparecer tentativas de formalizar as cadeias de serviços (BRADY e FELLENZ, 2007). Neste sentido, Ellram et al. (2004) propõem um modelo para as cadeias de prestação de serviços, baseados nas melhores práticas de modelos tradicionais para as cadeias de manufatura e adaptando-os ao fim desejado. Este modelo é apresentado na Figura 1, onde pode ser visualizado o destaque dado ao fluxo de informações nesta cadeia. 
Figura 1 - Modelo de cadeias de prestação de serviços

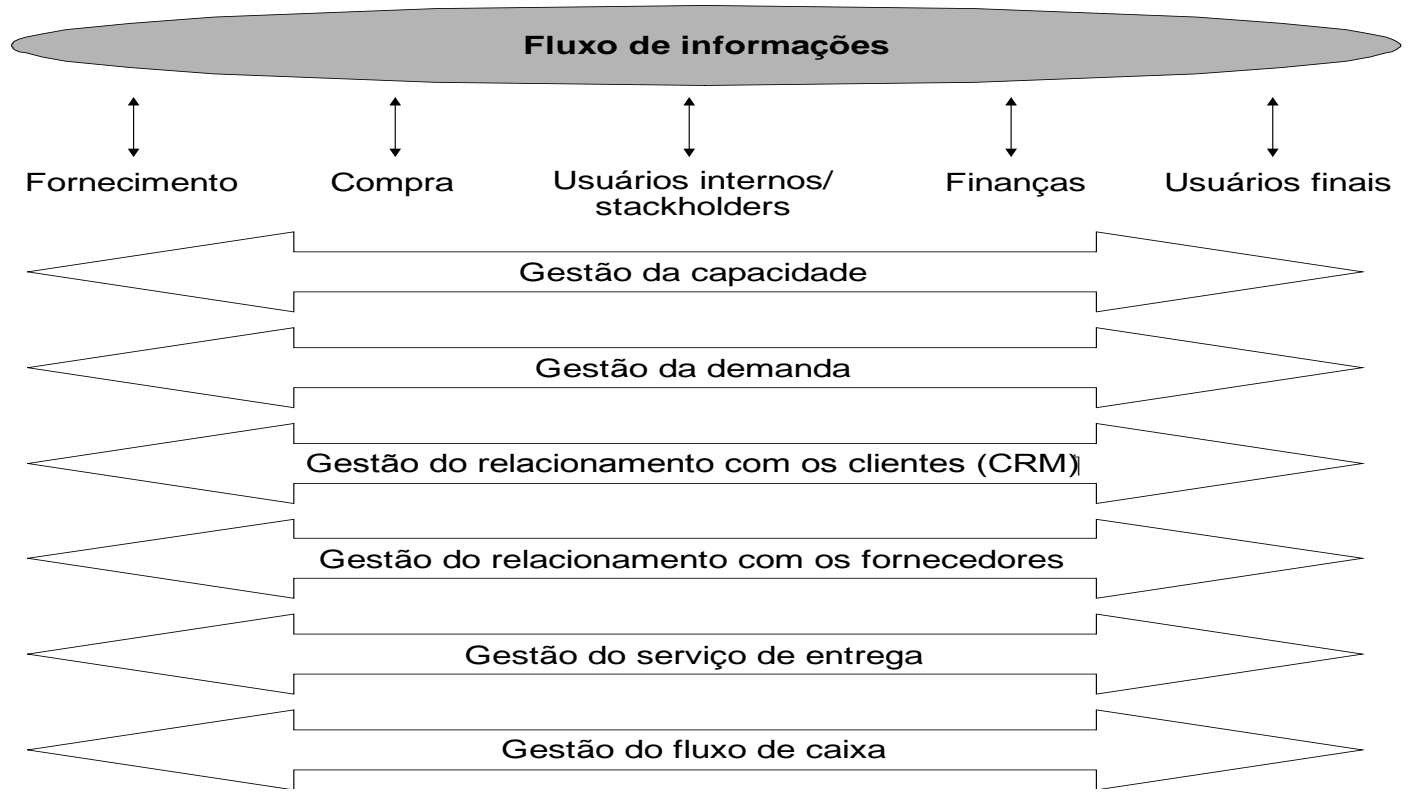

Fonte: Adaptado de Ellram et. al (2004).

Em um artigo convidado para a Revista Gestão\&Produção sobre as direções (agenda) de pesquisas em gestão de operações, Starr (2005) afirma que grande parte da economia das nações desenvolvidas é baseada na prestação de serviços, e que apesar disso, existem grandes oportunidades e desafios nesta área. $O$ autor apresenta o termo service supply chain, que pode ser livremente traduzido como cadeias de prestação de serviços ou cadeias de serviço, em contraponto às tradicionais cadeias de suprimentos, que poderiam ser identificadas como industriais. Akkermans e Vos (2003) também destacam a importância das cadeias de serviço e questionam se a gestão das ampliações das variações da demanda (conhecidas como Efeito Forrester, bullwhip ou chicote) também é válida nestas cadeias. Yang et al. (2004) apresentam um estudo de simulação de cadeias de suprimentos de serviços, definidas como a decomposição de um serviço, negociação com os fornecedores e prestação do serviço elo por elo. Um jogo para o ensino e pesquisa de cadeias de prestação de serviços é apresentado por Anderson Jr. e Morrice (2000), onde a gestão da capacidade das empresas é o principal desafio.

Os fatores ou práticas que influenciam em cadeias de suprimentos e de prestação de serviços foi analisado por Sengupta et al. (2006), que afirmam que a 
grande maioria dos estudos no setor são relacionados às cadeias de manufatura. Em suas conclusões apontam que as práticas de compartilhamento de informações influenciam significativamente e positivamente estas cadeias.

\section{MÉTODO DE PESQUISA}

Este artigo trata-se de um estudo exploratório sobre o gerenciamento da cadeia de suprimentos em um restaurante acadêmico, dentro do contexto de empresa júnior.

Segundo Cervo e Bervian (2002), os estudos exploratórios não elaboram hipóteses a serem testadas no trabalho, restringindo-se a definir objetivos e buscar mais informações sobre determinado assunto. Assim, recomenda-se o estudo exploratório quando há poucos conhecimentos sobre o problema a ser estudado, conforme objeto do presente estudo. Segundo Hart (1998), entre os objetivos da pesquisa exploratória está fornecer uma melhor compreensão do contexto e examinar a viabilidade de estudos futuros, indicando temas relevantes para serem estudados em maior profundidade.

Segundo Cooper e Schindler (2011), tanto as técnicas qualitativas quanto as quantitativas são aplicáveis em estudos exploratórios, embora a exploração se baseie mais nas técnicas qualitativas. Assim, optou-se pela utilização da técnica de pesquisa qualitativa.

\subsection{Unidade de análise}

De acordo com SANTOS et al. (2010), a descrição de uma cadeia de suprimentos deve ser feita a partir de uma empresa, denominada "empresa foco". Assim, para o presente trabalho selecionou-se o restaurante acadêmico da Universidade Federal de Itajubá (UNIFEI), por ser, até onde se conhece, o único restaurante estruturado como empresa júnior. 


\subsection{Coleta de dados}

Quando se considera o escopo da pesquisa qualitativa, diversas abordagens são adaptáveis das questões gerenciais para a pesquisa exploratória, entre elas: entrevistas exploratórias, observação e análise de documentos (COOPER e SCHINDLER, 2011). Estes foram os meios utilizados para a coleta de dados neste trabalho.

Foram realizadas duas entrevistas semiestruturadas, com duração aproximada de uma hora e meia cada. Para a condução da entrevista foi elaborado um roteiro com temas gerais a serem abordados e com perguntas específicas, quando havia a necessidade de uma exploração mais detalhada. Para aumentar a confiabilidade e a abrangência das informações, as entrevistas foram realizadas separadamente com uma dupla gerencial e com outra operacional ligada à área de compras e estoques, que como será discutido mais a frente, controla algumas das atividades mais importantes da empresa. Para aumentar a eficácia houve a confirmação dos dados e feedback com os entrevistados, conforme sugerido por Voss; Tsikriktsis e Frohlich (2002).

Cooper e Schindler (2011) afirmam que a observação direta é muito flexível, pois permite ao observador reagir e registrar aspectos sutis de fatos e comportamentos conforme eles ocorrem. Esta coleta foi conduzida através de uma visita à área de estoques da empresa e algumas visitas anônimas como consumidora.

Outra fonte de dados foram as informações disponíveis no sítio eletrônico da empresa e troca de e-mails com membros da organização.

As limitações da abordagem empregada neste trabalho relacionadas à subjetividade, não-representatividade e planejamento não sistemático foram ressaltadas por Cooper e Schindler (2011). Entretanto, como o presente estudo não tem como objetivo o teste de hipóteses, nem a generalização de um conceito e ao contrário, pretende confirmar ou não a importância do assunto, bem como levantar possíveis temas para maior aprofundamento, o presente método mostrou-se adequado.

Revista Produção Online, Florianópolis, SC, v.15, n. 1, p. 375-402, jan./mar. 2015. 


\section{RESULTADOS}

O Restaurante Acadêmico (RA) existe desde 2005 como uma proposta dos alunos participantes do Diretório Acadêmico da UNIFEI. Entretanto, em 2008 passou por uma série de mudanças e veio a se tornar a RA Empreendimentos Júnior, ou RA Júnior. A RA Empreendimentos Júnior foi idealizada para ser um laboratório de empreendedorismo da UNIFEI. O objetivo principal desta organização é desenvolver habilidades empreendedoras e criar um ambiente de fomento ao aprendizado para os alunos de graduação que participam do projeto.

O negócio principal da empresa é o "Restaurante Acadêmico", restaurante e lanchonete localizado dentro do campus. Contudo, pelo próprio ciclo de inovação e busca por oportunidades para suprir novas demandas, criou-se o departamento de Eventos, que é responsável por serviços de alimentação para eventos realizados dentro da universidade.

\subsection{Estrutura organizacional}

A administração da empresa é composta por conselheiros, quatro diretores e oito gerentes e a operação é executada por oito funcionários contratados em CLT, cerca de trinta funcionários-aluno, uma nutricionista e um contador terceirizados, totalizando, aproximadamente, cinquenta funcionários. O organograma da empresa é apresentado na Figura 2.

Figura 2 - Organograma da RA Empreendimentos Júnior

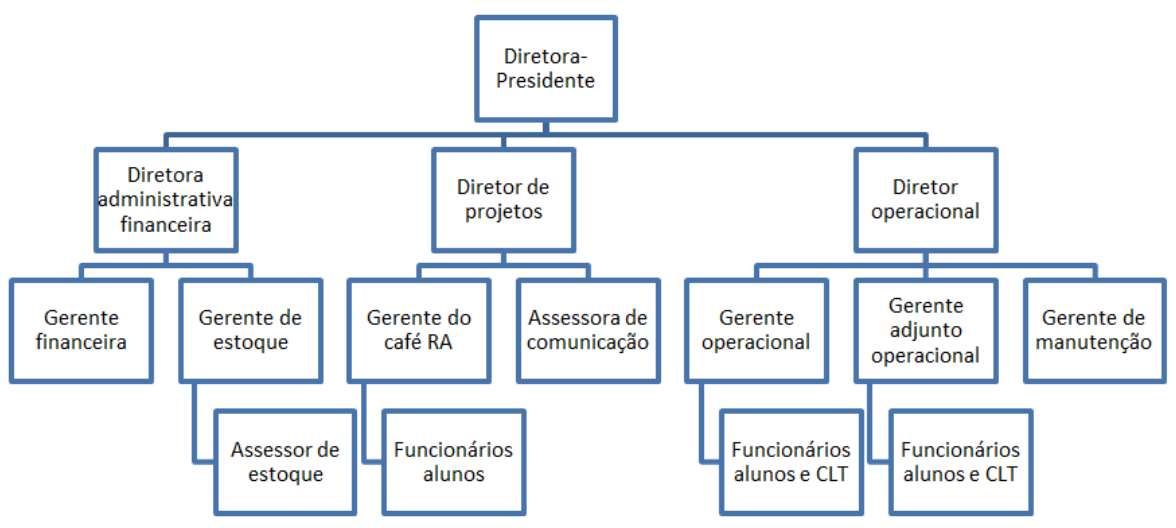

Revista Produção Online, Florianópolis, SC, v.15, n. 1, p. 375-402, jan./mar. 2015. 


\subsection{A cadeia de suprimentos}

Conforme discutido anteriormente, a cadeia de suprimentos engloba o fluxo de atividades, dinheiro e informação desde o fornecedor do fornecedor até o cliente do cliente, através de relações integradas e coordenadas entre seus membros, de modo a fornecer produtos e serviços, com o melhor nível de serviço possível ao cliente e ao menor custo possível. Assim, com o objetivo de mapear a cadeia de suprimentos do RA foram identificados, inicialmente, alguns elementos chaves: bens e serviços, fornecedores, clientes, atividades e processos e sistemas de informação.

\subsubsection{Bens e serviços}

Segundo Hoffman e Bateson (2003), a distinção entre bens e serviços nem sempre é perfeitamente clara. Os autores ressaltam que dificilmente é possível encontrar uma organização que se caracterize pelo fornecimento de bem puro ou serviço puro. Da mesma maneira, o RA como restaurante e lanchonete, caracterizase principalmente como uma empresa de serviços, porém apresenta também o fornecimento de alguns bens. Assim, o produto do RA caracteriza-se por oferta de serviços e bens.

Como a principal característica dos serviços é a intangibilidade, podemos citar como serviços principais do RA: o preparo de refeições e o preparo de salgados (desde o início de abril/2012) e o atendimento aos clientes e o serviço de acompanhamento nutricional. Tais serviços são enxergados pelo cliente através de características como: cordialidade no atendimento e qualidade, balanceamento e variedade das refeições e dos salgados e qualidade do serviço de acompanhamento nutricional e preço acessível e proximidade e facilidade de acesso e disponibilidade (horários de atendimento) e rapidez no atendimento e acessibilidade dos funcionários e predisposição a ajudar e entre outros.

Como oferta de bens, podemos citar: venda de bebidas, balas, chocolates, chips de celulares, entre outros produtos industrializados. Não há um controle rígido e padronizado sobre o número de bens comprados e preparados pela própria 
empresa, considerando seus diferentes tipos e variedades, porém, cada área realiza seu controle através de planilhas de Excel e/ou softwares, com agrupamentos diferentes, que variam conforme a utilidade para cada área.

\subsubsection{Fornecedores e clientes}

Considerando apenas o primeiro nível à montante, há cerca de vinte fornecedores fixos e alguns poucos esporádicos, conforme necessidade. De acordo com a responsabilidade pelo pedido, a forma de pedir e os dias de entrega, os fornecedores podem ser divididos em cinco grandes grupos, com alguns fornecedores podendo fazer parte de mais de um grupo. A saber: fornecedores de materiais para preparo das refeições (exceto carne e hortifruti), fornecedores de carne e hortifruti, fornecedores de salgados prontos, fornecedor de pão-de-queijo e fornecedores de produtos para a lanchonete (exceto salgados prontos).

Os clientes do RA são consumidores finais, que frequentam diariamente a empresa, como: alunos, professores e funcionários da Universidade. Entre consumidores esporádicos, tem-se: moradores da cidade e pais e familiares de alunos e participantes de eventos, como membros de empresas, organizações públicas, entre outros. Em média, são atendidas por dia cerca de quatrocentas a quinhentas pessoas no horário do almoço. Porém, não há uma estimativa precisa sobre o número de pessoas atendidas nos demais horários.

\subsubsection{Atividades e processos}

As unidades de restaurante e lanchonete são gerenciadas em conjunto. Estabeleceram-se três atividades como principais da empresa:

a) Preparação e oferta de refeições: a preparação das refeições é realizada por cozinheiras contratadas em regime de CLT, com base no cardápio preparado pela nutricionista. Tal atividade é complexa por lidar com alguns materiais perecíveis e com alta variabilidade de demanda.

b) Preparação e oferta de salgados: esta atividade iniciou-se com a implementação da fábrica de salgados no RA.

Revista Produção Online, Florianópolis, SC, v.15, n. 1, p. 375-402, jan./mar. 2015. 
c) Venda de produtos: esta atividade engloba, de maneira simplificada, a compra e revenda de produtos e sua maior dificuldade está na definição do ponto de ressuprimento para atender à demanda variável.

Como processos-chaves para um bom gerenciamento da cadeia de suprimentos foram considerados: a gestão do relacionamento com clientes (do inglês, Customer Relationship Management - CRM), a gestão do relacionamento com fornecedores (do inglês, Supplier Relationship Management - SRM) e a logística reversa da empresa.

a) Gestão do relacionamento com clientes:

Segundo Gordon (2002), a gestão do relacionamento com clientes é uma série de estratégias e processos que criam valores novos e mútuos para os clientes, estimula a preferência pela organização e melhora os resultados do negócio ao longo do relacionamento com o cliente. Para que isso seja possível, é importante entender, analisar e prever os desejos, expectativas e reações dos clientes.

Os membros da organização procuram avaliar a aceitação dos produtos (bens e serviços) pelos clientes através de conversas informais com colegas e demais alunos da Universidade. Em geral, observa-se que os produtos têm boa aceitação e, apesar da ausência de pesquisa estruturada, quando há uma baixa aceitação de um produto esta é facilmente identificada pelos funcionários da empresa e as devidas providências são tomadas (substituição do produto por outro, por exemplo).

Quanto à previsão de demanda, não há na organização registros de dados de vendas ou números de pessoas atendidas. Além disso, por experiência, já se constatou que a demanda da empresa é muito inconstante por conta de eventos (férias e feriados, palestras com coffee break, semanas de provas, etc.) e crescimento acelerado da Universidade. Tais eventos, muitas vezes não são de conhecimento da direção da empresa. Assim, devido à complexidade, alta variabilidade e ausência de registros, não é possível elaborar uma previsão de demanda adequada. A mesma é feita, então, através da experiência e conhecimento dos funcionários.

A pesquisa de aceitação dos produtos realizada pelos funcionários demonstra que a organização possui alguns processos de uma estrutura de gestão de 
relacionamento com os clientes, porém, por outro lado, a ausência de registros históricos de vendas demonstra que ainda há um longo caminho a ser percorrido para alcançar um nível de CRM que agregue valor tanto para o cliente, quanto para a empresa.

Para desenvolver um CRM mais efetivo, sugere-se, por exemplo, que a pesquisa de aceitação dos produtos seja realizada de uma maneira mais formal, de modo que seja possível atingir o maior número possível de consumidores. Além disso, é importante fazer o registro da mesma para consultas futuras, procurar registrar as vendas e ocorrências de falta de produto e disponibilizar um espaço para sugestões e reclamações dos clientes. Tais atividades são simples de serem implementadas e podem não só melhorar, como consolidar o relacionamento entre a empresa e os clientes.

b) Gestão do relacionamento com fornecedores:

De acordo com Lambert (2008), a gestão de relacionamento com fornecedores é o processo de gerenciamento da cadeia de suprimentos que provê a estrutura sobre como as relações com os fornecedores são desenvolvidas e mantidas.

Para desenvolver o SRM, ainda que de maneira tímida, a empresa conta com uma base pequena de fornecedores fixos, com os quais busca parcerias e uma base de fornecedores esporádicos para suprir eventuais demandas. O relacionamento é realizado na base da confiança e transparência. Como exemplo, pode-se citar situações como:

- Erros de entrega: quando há ocorrência de erros nas entregas, o recebimento é realizado em sua totalidade pelo RA e os erros são corrigidos pelos fornecedores na próxima entrega, sem prejuízo para o fornecedor ou para a empresa.

- Falta de produto por problemas de fornecimento: algumas vezes ocorre falta de algum produto por problemas de fornecimento, principalmente bebidas refrigerantes durante épocas festivas. Quando este problema ocorre, normalmente falta apenas certa variedade do produto. Para minimizar os efeitos desta falta para o cliente, a organização negocia descontos com os fornecedores para as variedades de produto disponíveis.

Revista Produção Online, Florianópolis, SC, v.15, n. 1, p. 375-402, jan./mar. 2015. 
- Alteração de quantidade pedida ou inclusão de novo item: quando há alta variação de demanda e necessidade de alterar a quantidade de um determinado item pedido ou incluir um novo item, a empresa entra em contato com o fornecedor por telefone (ou e-mail) e solicita a alteração da quantidade. Em geral, como a relação é de parceria, os fornecedores buscam atender às alterações solicitadas.

Entretanto, pode-se observar que o SRM ocorre com poucos registros oficiais. Para tornar o SRM um processo importante dentro da empresa, é importante realizar medições de desempenho dos fornecedores, bem como registro do número de solicitações de alterações realizadas e compartilhamento das informações com maior frequência.

c) Logística reversa:

Logística reversa é a estrutura responsável pelo gerenciamento do fluxo reverso de produtos: reciclagem, reuso, recall, devoluções, entre outros e tem se tornado um importante elemento do gerenciamento da cadeia de suprimentos (ROGERS e TIBBEN-LEMBKE, 2011).

A empresa apresenta algumas atividades como parte da estrutura de logística reversa. Entre elas:

- Resto ingesta: os restos de alimentos, chamados resto ingesta, são separados pela empresa para utilização por funcionários da UNIFEI para alimentação de animais.

- Outros resíduos: os demais resíduos são separados e a Cooperativa de Reciclagem de Itajubá os coleta periodicamente para reciclagem.

- Embalagem: alguns fornecedores entregam os produtos em caixas de papelão e as buscam posteriormente para reutilizá-las em novas entregas.

Além das atividades e processos mencionados acima, é importante analisar dentro do contexto do GCS as atividades de controle de estoque, compras e armazenagem:

- Controle de estoque e compras: esta pode ser considerada uma das atividades mais importantes da empresa, uma vez que não há previsão de demanda e os produtos tratados são, em sua maioria, perecíveis e as definições de quando comprar, quanto comprar, estoque de segurança, entre Revista Produção Online, Florianópolis, SC, v.15, n. 1, p. 375-402, jan./mar. 2015. 
outras são baseadas em "feeling" e no conhecimento dos membros da organização e, ao mesmo tempo, deseja-se manter um bom nível de serviço ao cliente, mas sem aumentar as sobras e desperdícios e, consequentemente, os custos. Normalmente os pedidos emitidos são pequenos e, por isso, não há conferência entre a documentação e o que foi pedido, havendo apenas conferência entre documentação e físico. É executado um controle do primeiro a entrar - primeiro a sair (PEPS), porém de forma manual e não há um procedimento de contagem de inventário estabelecido.

- Armazenagem: a empresa apresenta um único depósito, localizados nas dependências do restaurante/lanchonete. Desde abril de 2012, foi adotado lugar fixo para armazenamento de cada produto, com o objetivo de aplicar o 5 S e melhorar o processo.

\subsubsection{Sistemas de informação}

Os três softwares utilizados pela empresa são: Open Bar ${ }^{\circledR}, \mathrm{J}$ Finanças ${ }^{\circledR}$ e Excel®.

O Open Bar® é utilizado para registro de vendas e de retirada, abastecimento de dinheiro no caixa, fechamento de caixa, cadastro de novos clientes, abertura de comandas, alteração de preço do produto, controle de notas fiscais e relatórios. Neste software, há ainda a função de controle de estoque, porém a mesma não é utilizada pela empresa atualmente.

O J Finanças ${ }^{\circledR}$ é, segundo a própria empresa, pouco explorado, sendo utilizado apenas para controle de todos os valores pagos e recebidos.

O Exce/® é utilizado para controle de custos (inclusive rateio de custos de mão-de-obra entre o restaurante e a lanchonete), controle de entrada e saída do estoque, entre outros.

Nesta atividade, pôde-se observar baixa exploração e utilização dos recursos disponíveis, baixo registro das informações e, quando existe, pouco compartilhamento por todas as áreas e, por fim, ausência de definição de um fluxo 
de comunicação e informação entre os departamentos da empresa, de modo a tornar as informações disponíveis para todos.

As informações mencionadas acima encontram-se consolidadas no diagrama SIPOC (Suppliers, Inputs, Process, Outputs and Customers), conforme Figura 3. A pesquisadora optou pela utilização desta ferramenta para determinar os processos chaves e seus respectivos responsáveis dentro da cadeia de suprimentos da empresa e, assim, identificar oportunidades de melhoria e de estudos aprofundados, conforme objetivo da pesquisa.

Figura 3 - SIPOC dos processos-chaves da RA Empreendimentos Júnior

\begin{tabular}{|c|c|c|c|c|}
\hline $\begin{array}{l}\text { Fornecedores de } \\
\text { insumos e produtos } \\
\text { industrializados } \\
\text { Funcionários da } \\
\text { organização } \\
\text { Consumidores } \\
\text { finais (clientes) } \\
\text { Reitoria }\end{array}$ & $\begin{array}{l}\text { Matérias-primas } \\
\text { (came, verduras, } \\
\text { farinha, etc.) } \\
\text { Produtos } \\
\text { industrializados } \\
\text { (refrigerantes, } \\
\text { chocolate, balas, etc.) } \\
\text { Informações } \\
\text { Equipamentos } \\
\text { (fogão, freezer, } \\
\text { mesas, etc.) } \\
\text { Funcionários da } \\
\text { empresa } \\
\text { Energia e água }\end{array}$ & $\begin{array}{c}\text { Preparação e oferta } \\
\text { de refeições e } \\
\text { salgados e venda } \\
\text { de bebidas, doces, } \\
\text { etc. }\end{array}$ & $\begin{array}{c}\text { Refeições } \\
\text { Salgados } \\
\text { Doces } \\
\text { Bebidas } \\
\text { Cordialidade } \\
\text { Agilidade } \\
\text { Qualidade, } \\
\text { balanceamento e } \\
\text { variedade } \\
\text { Acompanhamento } \\
\text { nutricional } \\
\text { Custos } \\
\text { Disponibilidade } \\
\text { Facilidade de } \\
\text { acesso }\end{array}$ & $\begin{array}{c}\text { Alunos } \\
\text { Professores } \\
\text { Funcionários da } \\
\text { Universidade } \\
\text { Visitantes } \\
\text { (moradores da cidade, } \\
\text { parentes de alunos, } \\
\text { professores e } \\
\text { funcionários e } \\
\text { participantes de } \\
\text { eventos) }\end{array}$ \\
\hline
\end{tabular}

A partir do diagrama SIPOC, foi possível elaborar um fluxo sintetizado da cadeia de suprimentos, conforme Figura 4.

Revista Produção Online, Florianópolis, SC, v.15, n. 1, p. 375-402, jan./mar. 2015. 
Figura 4 - Fluxo da cadeia de suprimentos da RA Empreendimentos Júnior

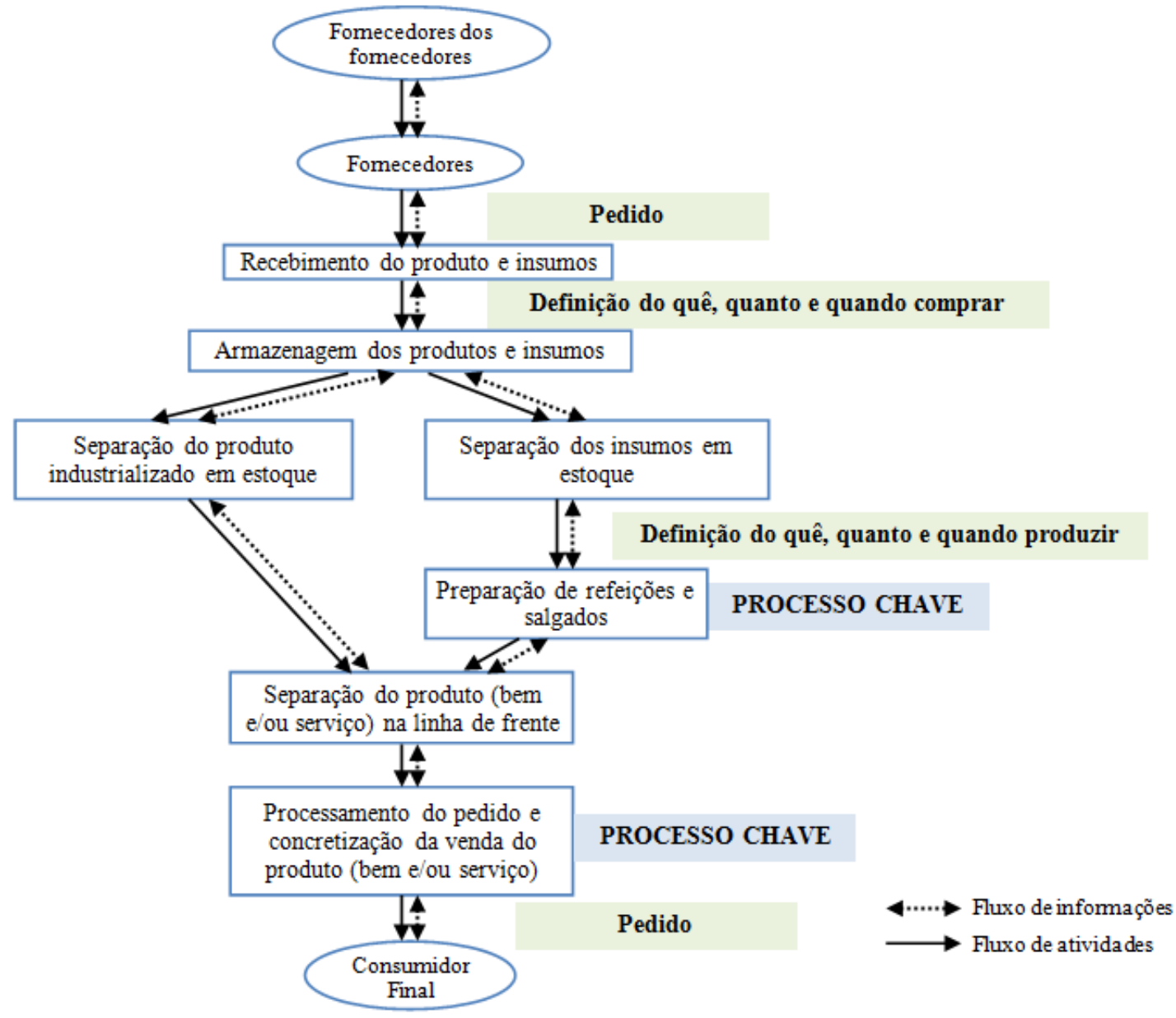

Com base nos levantamentos realizados, foi possível fazer a análise SWOT (Strengths, Weakness, Opportunities and Threats) da cadeia de suprimentos da empresa. A matriz SWOT é uma forma útil de analisar o ambiente organizacional, tornando possível verificar oportunidades para desenvolvimento de estudos mais profundos. O resultado de sua aplicação pode ser visto na Figura 5.

Revista Produção Online, Florianópolis, SC, v.15, n. 1, p. 375-402, jan./mar. 2015. 
Figura 5 - SWOT da cadeia de suprimentos da RA Empreendimentos Júnior

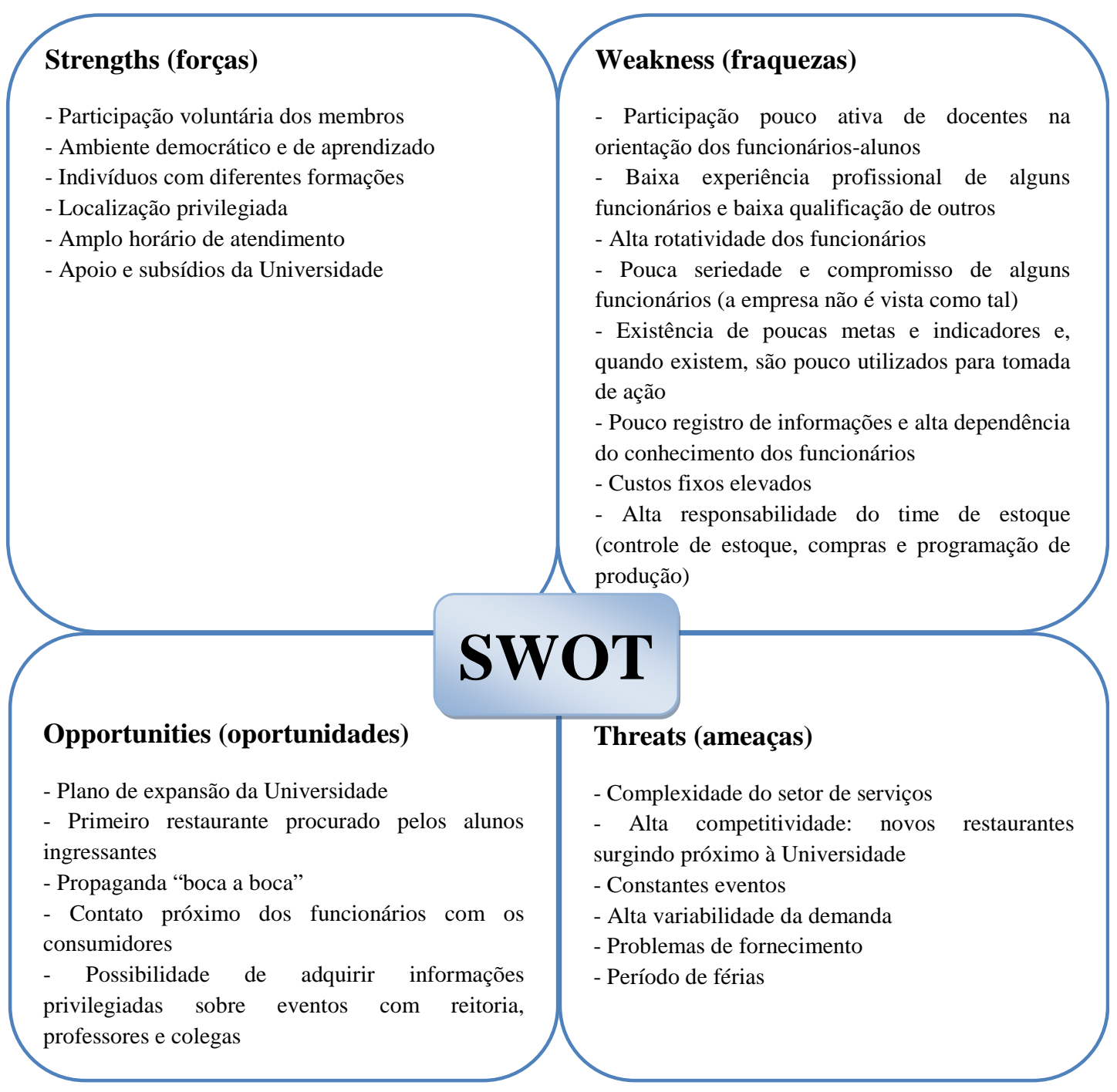

Com base no fluxo e na análise SWOT elaborada, verificou-se alguns temas relevantes para desenvolvimento de estudos mais profundos, como:

- Análise da variabilidade de demanda em restaurantes universitários (causa e efeito);

- Criação de modelos de previsão de demanda para restaurantes, no contexto de alta variabilidade e sazonalidade;

- Impactos da ausência de um produto em restaurantes universitários;

- Impactos da implementação de um novo processo complexo, mas que agrega valor ao cliente, como o caso da fábrica de salgados;

- A importância do controle de estoque em restaurantes universitários: não apenas como controle de custos, mas para agregar valor; Revista Produção Online, Florianópolis, SC, v.15, n. 1, p. 375-402, jan./mar. 2015. 
- Proposta de modelos de sistemas de informação voltados para restaurantes universitários;

- Aplicabilidade de tecnologia da informação, como o RFID, em pequenas empresas;

- Quais são os resultados quantitativos e qualitativos de um restaurante, estruturado como laboratório de empreendedorismo: tê-los ou não?

- Como é possível obter resultados sem métricas e indicadores: qualidade do líder, sorte ou há alternativas?

- A concentração de responsabilidades sob um setor é perigosa?

Entre outras questões que podem ser levantadas a partir deste trabalho. Tais estudos poderão auxiliar empresas, como a RA Empreendimentos Júnior, a gerar valor para o negócio de modo a se manterem competitivas no mercado.

\section{CONSIDERAÇÕES FINAIS}

Diante da situação mercadológica de intensa competitividade, constantes transformações e "comoditização" dos produtos e serviços, empresas de todos os segmentos buscam meios para aumentar sua qualidade, rapidez, confiabilidade e flexibilidade e, consequentemente, a satisfação de seus clientes e também de reduzir custos para possibilitar sua sobrevivência no mercado. O setor de serviços é um destes ramos. Tais fatores mostram a relevância de se desenvolver estudos voltados para a melhor compreensão, geração de teoria e comprovação de hipóteses relacionadas ao setor de serviços.

Assim, este trabalho traz como principal contribuição o desenvolvimento de uma melhor compreensão deste contexto e a análise da viabilidade de trabalhos futuros, propondo alguns temas relevantes para serem analisados com mais detalhamento.

Espera-se, ainda, que este artigo seja um estímulo para os pesquisadores brasileiros iniciarem estudos voltados para a área o mais breve possível, de modo a gerar teoria inédita e inovadora, reforçar a importância de projetos como o RA Empreendimentos Júnior e auxiliar para o aumento da competitividade e a continuidade dos mesmos.

Revista Produção Online, Florianópolis, SC, v.15, n. 1, p. 375-402, jan./mar. 2015. 


\section{AGRADECIMENTOS}

Os autores agradecem a Fundação de Amparo à Pesquisa do Estado de Minas Gerais (FAPEMIG), a Coordenação de Aperfeiçoamento de Pessoal de Nível Superior (Capes) e ao Conselho Nacional de Desenvolvimento Científico e Tecnológico (CNPQ) pelo apoio concedido a esta pesquisa.

\section{REFERÊNCIAS}

AKKERMANS, H. e VOS, B. Amplification in service supply chains: an exploratory case study from the telecom industry. Production and Operations Management, v. 12, n. 2, 2003. http://dx.doi.org/10.1111/j.1937-5956.2003.tb00501.x

ANDERSON Jr., E. G. e MORRICE, D. J. A simulation game for teaching service-oriented supply chain management: does information sharing help managers with service capacity decisions? Production and Operations Management, v. 9, n. 1, 2000. http://dx.doi.org/10.1111/j.1937-5956.2000.tb00322.x

BALLOU, R. H. Gerenciamento da cadeia de suprimentos/logística empresarial. 5. ed. Porto Alegre: Bookman, 2004.

BALTACIOGLU, T. e ADA, E. e KAPLAN, M. D e YURT, O. e KAPLAN, Y. C. A new framework for service supply chains. The Service Industries Journal, v. 27, n. 2, 2007. http://dx.doi.org/10.1080/02642060601122629

BERKLEY, B. J. e GUPTA, A. Identifying requirements to deliver quality service. International Journal of Service Industry Management, v. 6, n. 5, 1995. http://dx.doi.org/10.1108/09564239510101509

BERRAH, L. e CLIVILLE, V. Towards an aggregation performance measurement system model in a supply chain context. Computers in Industry, v. 58, n. 7, 2007. http://dx.doi.org/10.1016/i.compind.2007.05.012

BRADY, M., FELLENZ, M. Researching the role of information and communications technology (ICT) in contemporary marketing practices. Journal of Business \& Industrial Marketing, v. 23, n. 2, 2007. http://dx.doi.org/10.1108/08858620810850227

CERVO, A. L. e BERVIAN, P. A. Metodologia Científica. 5. ed. São Paulo: Prentice Hall, 2002.

CHILDERHOUSE, P. e HERMIZ, R. e MASON-JONES, R. e POPP, A. e TOWILL, D. R. Information flow in automotive supply chains: identifying and learning to overcome barriers to change. Industrial Management \& Data Systems, v. 103, n. 7, 2003 (a)

http://dx.doi.org/10.1108/02635570310489197

CHILDERHOUSE, P. e HERMIZ, R. e MASON-JONES, R. e POPP, A. e TOWILL, D. R. Information flow in automotive supply chains: present industrial practice. Industrial 
Management \& Data Systems, v. 103, n. 3, 2003 (b)

http://dx.doi.org/10.1108/02635570310465625

CHOU, M. C. e YE, H. e YUAN, X. M. e CHENG, Y. N. e CHUA, L. e GUAM, Y. e LEE, S. E. e TAY, Y. C. Analysis of a Software-Focused Products and Service Supply Chain. IEEE

Transactions on Industrial Informatics, v. 2, n. 4, 2006.

http://dx.doi.org/10.1109/TII.2006.885926

COOPER, D. R. e SCHINDLER, P. S. Métodos de Pesquisa em Administração. 10. ed. Porto Alegre: Bookman, 2011.

DROHOMERETSKI, E. e FAVARETTO, F. O impacto da contagem cíclica na acuracidade de estoque: múltiplos casos em indústrias do Paraná. Produção Online, v. 13, n. 2, 2013. http://dx.doi.org/10.14488/1676-1901.v13i2.1238

ELLRAM, L. M. e TATE, W. L. e BILLINGTON, C. Understanding and Managing the Services Supply Chain. Journal of Supply Chain Management, v. 40, n. 3, 2004.

http://dx.doi.org/10.1111/j.1745-493X.2004.tb00176.x

FORSLUND, H. e JONSSON, P. The impact of forecast information quality on supply chain performance. International Journal of Operations \& Production Management. v. 27, n.. 1, 2007. http://dx.doi.org/10.1108/01443570710714556

FREITAS, A. L. P. A qualidade em serviços no contexto da competitividade. Produção Online, v. 5, n. 1, 2005. http://dx.doi.org/10.14488/1676-1901.v5i1.321

FREITAS, L. M. e DINIZ, A. C. M. e LEITE, M. S. A. e VILLAR, A. M. Os reflexos do efeito chicote nos custos logísticos de estoque: o caso de uma empresa componente da cadeia de suprimento de bebidas. Produção Online, v. 10, n. 2, 2010. http://dx.doi.org/10.14488/16761901.v10i2.401

GANESHAN, R. e HARRISON, T. P. Penn State University. Introduction to supply chain management. Penn State, 1995. Disponível em: <

http://mason.wm.edu/faculty/ganeshan r/documents/intro supply chain.pdf> Acesso em: 15 de setembro 2013.

GAVIRNENI, S. Information flows in capacitated supply chains with fixed ordering costs. Management Science, v. 48, n. 5, 2002. http://dx.doi.org/10.1287/mnsc.48.5.644.7806

GORDON, I. Best practices: customer relationship management. Ivey Business Journal, Vol. 67, nov./dec, 2002.

GRÖNROOS, C. Marketing: gerenciamento e serviços. 2. ed. Rio de Janeiro: Campus Elsevier, 2004.

GUNDLACH, G. T. e BOLUMOLE, Y. A. e ELTANTAWY, R. A. e FRANKEL, R. The changing landscape of supply chain management, marketing, channels of distribution, logistics and purchasing. The Journal of Business \& Industrial Marketing, Vol. 1, No. 7 , 2006. http://dx.doi.org/10.1108/08858620610708911

GUT, C. e BELFIORE, P. Um modelo de decisão para o uso de entreposto aduaneiro. Produção Online, v. 9, n. 1, 2009. http://dx.doi.org/10.14488/1676-1901.v9i1.204

Revista Produção Online, Florianópolis, SC, v.15, n. 1, p. 375-402, jan./mar. 2015. 
HART, C. Doing a Literature Review: Releasing the Social Science Research Imagination. 1. ed. London: SAGE Publications, 1998.

HOFFMAN, K. D. e BATESON, J. E. G. Princípios de marketing de serviços: conceitos, estratégias e casos. 1. ed. São Paulo: Pioneira Thomson Learning, 2003.

INSTITUTO BRASILEIRO DE GEOGRAFIA E ESTATÍSTICA. Classificação nacional de atividade econômica: relatório. Rio de Janeiro, versão 2, p.1-423, 2007.

INSTITUTO BRASILEIRO DE GEOGRAFIA E ESTATÍSTICA. Pesquisa anual de serviços: relatório. Rio de Janeiro, v. 10, p.1-205, 2008.

JANAK, S. The importance of information flow within the supply chain. Logistics

Information Management, v. 9, n.4, 1996. http://dx.doi.org/10.1108/09576059610123132

KÄRKKÄINEN, M. e ALA-RIKU, T. e FRÄMLING, K. The product centric approach: a solution to supply network information management problems? Computers in Industry, v. 52, 2003. http://dx.doi.org/10.1016/S0166-3615(03)00086-1

LAMBERT, D. Supply Chain Management: processes, partnerships, performance. 3. ed. Sarasota: Supply Chain Management Institute, 2008.

LEWIS, I. e TALAYEVSKY, A. Improving the interorganizational supply chain through optimization of information flows. Journal of Enterprise Information Management, v. 17,n. 3, 2004. http://dx.doi.org/10.1108/17410390410531470

LIN, C. e TSENG, H. Identifying the pivotal role of participation strategies and information technology application for supply chain excellence. Industrial Management \& Data Systems, v. 106, n. 5, 2006. http://dx.doi.org/10.1108/02635570610666476

LUMMUS, R. R. e VOKURKA, R. J. Defining supply chain management: a historical perspective and practical guidelines, Industrial Management \& Data Systems. v. 99, n. 1, 1999. http://dx.doi.org/10.1108/02635579910243851

MCLAUGHLIN, J. e MOTWANI, J. e MADAN, M. S e GUNASEKARAN, A. Using information technology to improve downstream supply chain operations: a case study. Business

Process Management Journal. v. 9, n. 1, 2003.

http://dx.doi.org/10.1108/14637150310461413

MINISTÉRIO DE DESENVOLVIMENTO, INDÚSTRIA E COMÉRCIO EXTERIOR (Brasil). 0 setor de serviços brasileiro. 2010. Disponível em:

$<$ http://www.mdic.gov.br/sitio/interna/interna.php?area=4\&menu=3123>. Acesso em: 9 de setembro 2013.

PIRES, S. R. I. e CARRETERO-DÍAZ, L. E. Gestión de la cadena de suministros. McGraw Hill, Madrid, 2007.

ROGERS, D. S. e TIBBEN-LEMBKE, R. An examination of reverse logistic practices. Journal of Business Logistics, v. 22, n. 2, 2001. http://dx.doi.org/10.1002/j.21581592.2001.tb00007.x

ROSSIN, D. An exploratory analysis of information quality in supply chains: efficient and responsive models. Journal of Global Business Issues, v. 1, n. 2, 2007.

Revista Produção Online, Florianópolis, SC, v.15, n. 1, p. 375-402, jan./mar. 2015. 
RUGGLES, K. Technology and the Service Supply Chain. Supply Chain Management Review, v. 9, n. 7, 2005.

SAHIN, F. e ROBINSON, E. P. Flow coordination and information sharing in supply chains: Review, implications and directions for future researches. Decision Sciences, v. 33, n. 4, 2002. http://dx.doi.org/10.1111/j.1540-5915.2002.tb01654.x

SANTOS, C. M. S. e LEITE, M. S. A. e LUCENA, A. D. e GRILO JÚNIOR, T. F. Evoluindo da cadeia de valor para cadeia de suprimentos. Produção Online, v. 10, n. 4, 2010. http://dx.doi.org/10.14488/1676-1901.v10i4.402

SCHNETZLER, M. J. e SCHÖNSLEBEN, P. The contribution and role of information management in supply chains: a decomposition-based approach. Production Planning \& Control, v. 18, n. 6, 2007. http://dx.doi.org/10.1080/09537280701499700

SENGUPTA, K. e HEISER, D. R. e COOK, L. S. Manufacturing and Service Supply Chain Performance: A Comparative Analysis. Journal of Supply Chain Management, v. 42, n. 4, 2006. http://dx.doi.org/10.1111/j.1745-493X.2006.00018.x

SIGALA, M. e CHRISTOU, E. Global trends and challenges in services. Managing Service Quality, v. 16, n. 4, 2006. http://dx.doi.org/10.1108/09604520610675685

SIMCHI-LEVI, D. e KAMINSKY, P. e SIMCHI-LEVI, E. Designing and managing the supply chain: concepts, strategies \& case studies. 2. ed. New York: McGraw-Hill/Irwin, 2003.

STARR, M. K. Changing agendas for operations management. Gestão\&Produção, v. 12, n. 13, 2005. http://dx.doi.org/10.1590/S0104-530X2005000300003

SWARTZ, N. Data Management Problems Widespread. The Information Management Journal, v 41, n. 5, 2007.

TORRES JÚNIOR, N. e MIYAKE, D. I. Melhoria de desempenho em serviços: alternativas para lidar com o trade-off entre eficiência e eficácia. Produção Online, v. 11,n. 1, 2011. http://dx.doi.org/10.14488/1676-1901.v11i1.539

TORRES JÚNIOR, N. e LOPES, A. L. M. A produtividade em serviços: uma análise à luz da revisão sistemática de literatura. Produção Online, v. 13, n. 1, 2013.

http://dx.doi.org/10.14488/1676-1901.v13i1.1164

VOSS, C e TSIKRIKTSIS, N e FROHLICH, M. Case research in operations management. International Journal of Operations \& Production Management, v. 22, n. 2, p. 195-219, 2002. http://dx.doi.org/10.1108/01443570210414329

WAART, D. e KEMPER, S. 5 steps to service supply chain excellence. Supply Chain Management Review, v. 8, n. 1, 2004.

WESTERBACK, L. K. Toward Best Practices for Strategic Information Technology Management. Government Information Quarterly, v. 17, n. 1, 2000. http://dx.doi.org/10.1016/S0740-624X(99)00023-4 
YANG, Z. e ZHANG, D. e XU, J. The simulation of service supply chain formation based on mobile agent's searching. Annals of the IEEE International Conference on E-Commerce Technology for Dynamic E-Business, 2004. http://dx.doi.org/10.1109/CEC-EAST.2004.66

ZENG, Y. e CHIANG, R. H. L. e YEN, D. C. Enterprise integration with advanced information technologies: ERP and data warehousing. Information Management \& Computer Security, v. 11, n. 3, 2003. http://dx.doi.org/10.1108/09685220310480390

ZHOU, H. e BENTON Jr. Supply chain practice and information sharing. Journal of Operations Management. v. 25, n. 6, 2007. http://dx.doi.org/10.1016/j.jom.2007.01.009

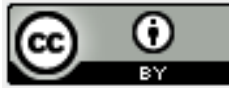

Artigo recebido em 29/11/2014 e aceito para publicação em 11/12/2014 DOI: http://dx.doi.org/ 10.14488/1676-1901.v15i1.1924 